Itinéraires Itinéraires

Littérature, textes, cultures

2020-3 | 2021

Le rap, une poésie de performances

\title{
Le rap, une poésie de performances
}

Présentation du numéro

\section{Magali Nachtergael}

\section{OpenEdition}

Journals

Édition électronique

URL : https://journals.openedition.org/itineraires/9369

DOI : 10.4000/itineraires.9369

ISSN : 2427-920X

Éditeur

Pléiade

Référence électronique

Magali Nachtergael, «Le rap, une poésie de performances », Itinéraires [En ligne], 2020-3 | 2021, mis en ligne le 11 décembre 2021, consulté le 12 décembre 2021. URL : http://journals.openedition.org/ itineraires/9369; DOI : https://doi.org/10.4000/itineraires.9369

Ce document a été généré automatiquement le 12 décembre 2021.

\section{(c) (i) $\Theta$}

Itinéraires est mis à disposition selon les termes de la licence Creative Commons Attribution - Pas d'Utilisation Commerciale - Pas de Modification 4.0 International. 


\title{
Le rap, une poésie de performances
}

\author{
Présentation du numéro
}

\section{Magali Nachtergael}

1 Le morceau de rap qui a fait connaître le genre, alors naissant, au grand public, Rapper's Delight de Sugarhill Gang, a fêté ses quarante ans en 2019. Depuis, le rap a connu le succès que l'on sait et est devenu l'une des productions de textes poétiques les plus importantes à l'échelle mondiale. Si l'on devait consigner tous les textes de rap dans une anthologie, il faudrait déjà plusieurs volumes. Mais est-il souhaitable de coucher ces textes sur du papier et de les y placer bien sagement ? N'est-il pas le propre du rap d'être une poésie performée, profondément rattachée à l'oralité ?

2 Apparue dans les années 1970, cette poésie performée est désormais démultipliée par les nombreux courants, ramifications et vedettes qui ont contribué à sa popularité actuelle. Analysé depuis longtemps en France par les sociologues (Béthune 2003, Pecqueux 2007, Hammou 2012) comme une expression culturelle à part entière, le rap, malgré des travaux, reste marginal dans les études littéraires contemporanéistes en France. Le slam, en revanche, grâce aux travaux de Camille Vorger (2010) notamment, profite d'une meilleure reconnaissance institutionnelle.

3 La question de la performance poétique est elle-même récente dans le champ des études littéraires (Penot-Lacassagne et Théval 2016) et mérite d'être elle-même pluralisée, en particulier dans le rap. D'abord, la performance du texte se double d'une performance musicale et l'ethos du rappeur (qui ne se déclare pas toujours poète au premier chef) se performe lui aussi dans un écosystème non pas éditorial mais scénique et désormais aussi numérique. Ensuite, la performance visuelle participe, en tant que médium, à sa diffusion et à sa forme. En effet, le rap est accompagné d'une mise en images par les clips, une dimension essentielle de son esthétique. Enfin, la performance sociale qu'impliquent les textes, soit dans la culture de la joute verbale et du clash (Vettorato 2008), soit dans la culture capitaliste ${ }^{1}$, se retrouve encore dans la revendication politique qui joue de son esthétique « hors la loi » (Béthune 2003, Ghio 2016).

4 Au-delà de ces considérations générales liées à la perception et la réception du rap, à l'intérieur même de ce champ comme champ esthétique, poétique et politique, des 
tensions de genre, de hiérarchie et de luttes articulent cet espace poétique d'affinités ou oppositions franches. Le rap en cela est un miroir de la contemporanéité, au même titre que l'art contemporain, dans la mesure où il engage une relation active avec son temps en faisant œuvre de commentaire, dénonciation, provocation ou appropriation, tout en touchant un public quantitativement bien plus important. Il est aussi une production culturelle située dans un contexte défini avec ses publics, ses lieux de performances et de diffusion. L'espace créatif du rap s'étend ainsi de la culture populaire à des médias comme le cinéma, avec des prédilections pour les films d'arts martiaux ou de super-héros, ou à des univers médiatiques associés au luxe, à la consommation et à une économie libidinale outrancière. Il performe également des tensions de genre (rap féminin vs masculin, performance de la masculinité ou de la féminité, cf. Ghio 2020) et de sociabilité (affirmations des origines, antiracismes, identités postcoloniales mais aussi labels et réseaux). Il développe enfin une culture visuelle à travers les clips qui déplacent le texte vers le scénario, jusqu'à la production de courts métrages qui témoignent d'un véritable travail de narration de type cinématographique à l'arrière-plan.

Ce volume se propose de relier la pratique du rap à l'univers des performances poétiques au sens large mais aussi de montrer en quoi cette poésie mise en rythme et en musique opère spécifiquement suivant le mode de la performance : morceaux de bravoure (freestyle, flow), ethos - persona - du rappeur, voire mythologie individuelle savamment construite, textes performatifs visant à libérer les individus de situations sociales qu'ils dénoncent, clips montrant un univers rêvé (ou cauchemardé, comme dans California Love de 2Pac feat. Dr. Dre). Organisé en trois parties, ce dossier présente des approches qui, souvent, se croisent et se recoupent. Nous avons choisi de les regrouper selon les aspects les plus saillants de la performance que les contributions mettaient en avant.

\section{Performance audiovisuelle et persona : identité, corps et visibilité}

6 La première partie rassemble les contributions autour de la performance audiovisuelle, qui, si elle est indissociable du texte, met en avant des stratégies liées à la persona de l'artiste comme le montre Charlotte Gagné-Dumais avec les rappeurs étatsuniens Ice Cube, Vince Staples et Tyler, the Creator en analysant les modes d'apparition de leurs identités multiples. Ces façons de fabriquer des identités par les clips permet évidemment d'interroger les représentations liées à la couleur de peau, l'origine et la situation sociale du rappeur ou de la rappeuse. Chez Casey, rappeuse franco-antillaise, Emily Schuman souligne l'émergence d'un discours critique sur les positions colonialistes françaises en formulant l'hypothèse de « l'écoute et la racialisation comme phénomènes audiovisuels " autour de la chanson "Créature ratée ", dont le clip a été réalisé par Chris Macari, connu également pour avoir élaboré la signature visuelle des clips de Booba. L'association de la représentation de l'identité et de son inscription dans le corps social est au cœur de l'article d'Elsa Vallot qui s'attache à la figure du rappeur hardcore à partir d'exemples français, notamment Kalash Criminel, Médine et Fianso avec la constellation du collectif 93 Empire. Dans un rapport agonistique entre une persona construite autour de l'univers de l'illicite et de la criminalité et la polis, vécue comme répressive par le seul prisme régalien de la police et de la justice (ou 
plutôt injustice), la spectacularisation du corps et la rhétorique belliqueuse prennent une dimension politique.

\section{Performances poético-politiques : des discours situés}

7 Peut-on parler d'une " poétique politique » de la performance pour des rappeurs ou des rappeuses? Leur modalité d'apparition dans les médias visuels et sonores montre que le lien entre création poétique et discours politique se performe dans un espace public élargi. Réciproquement, et c'est l'enjeu de l'article de Jovensel Ngamaleu qui aborde des textes de rappeurs masculins français (Booba, La Fouine) et camerounais (Valsero et Maalhox), le rap poursuit une fonction de réalisme social, en se donnant le rôle de révéler et dénoncer les aspects les plus cruels de la société, du racisme à l'économie sexuelle en passant par la corruption. Cette dimension politique peut être directement revendiquée par les rappeurs et renouveler la fonction et la position de l'engagement littéraire, ce que Lefranc Joseph et Vinson Bradley Noël ont analysé à partir d'une étude de terrain et des entretiens menés en Haïti autour du rap créole, à l'intersection entre pratique culturelle globalisée et discours situé dans un contexte politique et social défini. La performance s'entend donc aussi d'un point de vue de la poéticité, en lien avec une contemporanéité sociale. Denis Saint-Amand, en prenant appui sur les textes du groupe bordelais Odezenne, fait contraster la tradition chansonnière des mauvais garçons avec un «style potache » qui dénonce par le biais de la dérision et s'engage dans le contexte d'une autre actualité sociale contemporaine, la crise dite des «gilets jaunes » en France entre 2018 et 2019.

\section{Performance intermédiale et mémoire littéraire}

Photographie sociale, poésie agonistique, le rap performe et transforme aussi des traditions littéraires par des effets d'« intertextualité performée », selon les termes de Nina Rolland. La dernière partie du volume traite plus directement de notions littéraires connues, telles que l'intertextualité, et de prosodie. Cependant, Nina Rolland, à partir d'une étude sur l'héritage de Baudelaire dans le rap francophone, et Serigne Seye, autour d'une reprise de textes de Léopold Sédar Senghor, montrent que l'intermédialité constitutive du rap induit des manières de réactiver, de transformer la mémoire littéraire et de la projeter dans des espaces de réception qui traversent les frontières symboliques. Ce dont témoignent les deux articles, c'est que l'intégration de textes historiques implique une véritable re-création mais aussi une relecture de l'œuvre originale par son intégration dans le contexte d'une poétique populaire et intermédiale. Aussi, l'influence africaine-américaine sur les textes de Senghor devient plus prégnante à l'écoute autant que l'appropriation de cette figure historique inscrit le rap dans une célébration très explicite de la négritude. Le choix de citations de Baudelaire n'est pas non plus anodin, que ce soit pour se démarquer d'une tradition littéraire scolaire ou pour revendiquer aussi les attributs de l'archétype du poète maudit, écrivain du spleen et des rêveries mélancoliques. Mais il s'agit aussi de se positionner par rapport à une tradition par des métadiscours - que ce soit par la reprise directe ou par une transformation - tout en se mesurant aussi à cette tradition pour la défier et affirmer sa propre identité poétique. Julien Mowang analyse pour sa part la résurgence et l'omniprésence de la mémoire coloniale dans les textes de Médine, 
rappeur français d'origine algérienne, dont les chansons se font récit alternatif de l'histoire officielle.

\section{Performances orales et fabrique des singularités sonores}

9 L'oralité du rap, dernier aspect qu'aborde le volume, s'inscrit dans une ultime performance vocale et rythmique qui participe de l'ethos poétique du rappeur ou de la rappeuse. Le morceau de bravoure et sa signature sonore font partie des codes rapologiques qui réactivent aussi la dimension orale première de poésie. Léopold Reignier et Idir Mahiou analysent les particularismes du flow, terme qui désigne la profération rythmée des textes mis en beat et en musique. En comparant les pratiques syntaxiques et rythmiques du groupe historique étatsunien Wu-Tang Clan et celles du rappeur français Booba, Léopold Reignier invite à pratiquer le «close listening » calqué sur la méthode du « close reading » qui a structuré une tendance importante des études littéraires notamment dans le domaine stylistique. C'est d'un point de vue plus historique qu'Idir Mahiou analyse le flow selon une distinction entre flow binaire et " polyrythmique ", et transpose des outils d'analyse littéraire réservés à la lecture vers une écoute active des textes. Leurs contributions montrent comment les langues, les influences et la volonté de groupes de rappeurs de se distinguer, comme Dee Nasty, IAM et Ärsenik entre autres, ont travaillé à élaborer des signatures phonique, phonétique et rythmique singulières qui tracent une histoire des performances orales propres au rap.

\section{Le rap, au cœur de la création transmédiale et néolittéraire contemporaine}

10 L'univers du rap, s'il est un espace de présentation de soi au sens goffmanien, est aussi un univers contre-poétique : il s'oppose à des clichés de la poésie, douce à l'oreille, mais il produit lui-même des formes imaginaires liées tantôt à la puissance, la performance physique (par la danse, le virilisme, la sculpture des corps). Son agentivité dépasse de loin les cercles de la poésie de performance par les moyens de publication, médiatisation, communication et de diffusion sur lesquels il s'appuie : en prenant le virage du tournant visuel de la littérature, il relève à bien des égards d'une néolittérature qui se joue hors du livre et se performe par des outils technologiques plurimédiatiques mêlant texte, son et image. Il procède aussi à une performance sociale par la maîtrise de l'environnement urbain, imaginaire de la sauvagerie, et une conquête des espaces symboliques par des pratiques créatives qui relèvent aussi de la poésie, de la musique et des arts visuels. Le but n'est pas, ici, de muséifier le rap mais de mieux comprendre en quoi il fait directement écho à notre contemporanéité créative et littéraire, et à ses fantasmes de performance, dans un monde capitaliste où persistent de criantes inégalités.

11 Le rap confronte, au même titre que les littératures néomédiatiques, les études littéraires à ses propres outils d'analyse et à ses méthodologies. Ce numéro entend contribuer à élaborer une méthodologie adaptée, propre à l'étude du hip-hop et du rap, dans une perspective esthétique, transmédiale et liée aux performance studies. Il s'agit d'adapter et développer une approche critique intégrant des outils d'autres disciplines 
(anthropologie, études visuelles, esthétique, analyse du discours, sociolinguistique, sociologie, etc.) pour mettre en valeur une forme de création poétique encore peu intégrée aux corpus canoniques en raison de barrières symboliques.

\section{BIBLIOGRAPHIE}

Béthune, Christian, 2003, Le Rap, une esthétique hors-la-loi, Paris, Autrement, coll. « Mutations ». Béthune, Christian, 2004, Pour une esthétique du rap, Paris, Klincksieck, coll. « 50 questions ».

Freitas, Franck, 2011, “"Blackness à la demande” », Volume!, nº 8, vol 2, en ligne : http:// journals.openedition.org/volume/2696.

Ghio, Bettina, 2016, Sans fautes de frappe : rap et littérature, Marseille, Le mot et le reste.

Ghio, Bettina, 2019, Pas là pour plaire, Marseille, Le mot et le reste.

Hammou, Karim, [2012] 2014, Une histoire du rap en France, Paris, La Découverte.

Pecqueux, Anthony, 2007, Voix du rap. Essai de sociologie de l'action musicale, Paris, L'Harmattan, coll. « Anthropologie du monde occidental ».

Pecqueux, Anthony, 2009, Le rap, Paris, Cavalier Bleu, coll. « Idées reçues ».

Penot-Lacassagne, Olivier et Théval, Gaëlle (dir.), 2018, Poésie et performance, Nantes, ENCD.

Vettorato, Cyril, 2008, Le monde où l'on clashe, la joute verbale d'insultes dans la culture de rue, Paris, éd. Archives contemporaines.

Vicherat, Mathias, 2001, Pour une analyse textuelle du rap français, Paris, L'Harmattan.

Vorger, Camille, 2010, Slam, une poétique. De Grand Corps Malade à Boutchou, Valenciennes, Presses universitaires de Valenciennes, coll. «Cantologie ».

\section{NOTES}

1. Voir l'intervention de Franck Freitas au séminaire Fight the power, «Booba et la mise en traduction d'un esprit du capitalisme », jeudi 8 novembre 2018, séminaire CRESSPA, Emmannuelle Carinos, Keivan Djavadzadeh et Karim Hammou (dir.), Paris, 2018-2019. 


\section{AUTEUR}

MAGALI NACHTERGAEL

Université Bordeaux Montaigne, Plurielles (UR 24142) 